\title{
Anomalous net-baryon-rapidity spectra at RHIC
}

\section{Georg Wolschin ${ }^{1}$}

\author{
Institut für theoretische Physik der Universität, D-69120 Heidelberg, Germany
}

(Submitted January 02, 2003)

\begin{abstract}
Net-baryon rapidity distributions in central $\mathrm{Au}+\mathrm{Au}$ collisions at $\sqrt{s_{N N}}=200 \mathrm{GeV}$ are shown to consist of three components. The nonequilibrium contributions are accounted for in a Relativistic Diffusion Model. Near midrapidity, a third fraction containing $Z_{e q} \simeq 22$ protons reaches local statistical equilibrium in a discontinuous transition. It may be associated with a deconfinement of the participant partons and thus, serve as a signature for Quark-Gluon Matter formation.
\end{abstract}

\footnotetext{
${ }^{1}$ E-mail: wolschin@uni-hd.de
}

http://wolschin.uni-hd.de 
Rapidity distributions of participant (net) baryons are very sensitive to the dynamical and statistical properties of nucleus-nucleus collisions at high energies. Recent results for net-proton rapidity spectra in central $\mathrm{Au}+\mathrm{Au}$ collisions at the highest $\mathrm{RHIC}$ energy of $\sqrt{s_{N N}}=200 \mathrm{GeV}$ show an unexpectedly large rapidity density at midrapidity. The BRAHMS collaboration finds [1] $\mathrm{dN} / \mathrm{dy}=7.1 \pm 0.7$ (stat.) \pm 1.1 (sys.) at $\mathrm{y}=0$.

The $\Lambda, \bar{\Lambda}$ feed-down corrections reduce this yield by 17.5 per cent [1] when performed in accordance with the PHENIX $\Lambda$-results [2] at $130 \mathrm{GeV}$, but the amount of stopping remains significant, although a factor of about 4 smaller as compared to $\mathrm{Pb}+\mathrm{Pb}$ at the highest SPS energy. (A corresponding STAR result [3] for $\mathrm{y}=0$ at $130 \mathrm{GeV}$ does not yet include the feed-down correction). Many of the available numerical microscopic models encounter difficulties to predict the net-proton yield in the central midrapidity valley of the distribution, together with the broad peaks at the detected positions.

In this Letter I propose to interpret the data in a nonequilibrium-statistical Relativistic Diffusion Model. The net baryon rapidity distribution at RHIC energies emerges from a superposition of the beam-like nonequilibrium components that are broadened in rapidity space through diffusion due to soft (hadronic, low $p_{\perp}$ ) collisions and particle creations, and a statistical equilibrium (thermal) component at midrapidity that arises from hard (partonic, high $p_{\perp}$ ) processes.

At RHIC energies, the underlying distribution functions turn out to be fairly well separated in rapidity space. Since the transverse degrees of freedom are in (or very close to) thermal equilibrium, they are expected to decouple from the longitudinal ones. The time evolution of the distribution functions is then governed by a Fokker-Planck equation (FPE) in rapidity space [4, 5, 6, 7, 8, 9]. In the more general case of nonextensive (non-additive) statistics [10] that accounts for long-range interactions and violations of Boltzmann's Stosszahlansatz [6, 7, 12] as well as for non-Markovian memory (strong coupling) effects [11, 12, the resulting FPE for the rapidity y in the center-of-mass frame is

$$
\frac{\partial}{\partial t}[R(y, t)]^{\mu}=-\frac{\partial}{\partial y}\left[J(y)[R(y, t)]^{\mu}\right]+D(t) \frac{\partial^{2}}{\partial y^{2}}[R(y, t)]^{\nu}
$$

Since the norm of the rapidity distribution has to be conserved, $\mu=1$ is required, and the 
nonextensivity parameter that governs the shape of the power-law equilibrium distribution becomes $\mathrm{q}=2-\nu[10]$. In statistical equilibrium, transverse mass spectra and transverse momentum fluctuations in relativistic systems at SPS-energies $\sqrt{s_{N N}}=17.3 \mathrm{GeV}$ require values of $\mathrm{q}$ very slightly above one, typically $\mathrm{q}=1.038$ for produced pions in $\mathrm{Pb}+\mathrm{Pb}[10]$. For $q \rightarrow 1$, the equilibrium distribution converges to the exponential Boltzmann form, whereas for larger values of $\mathrm{q}$ (with $q<1.5$ ) significantly broader equilibrium distributions are obtained, and the time evolution towards them becomes superdiffusive [10, 13].

To study rapidity distributions in multiparticle systems at RHIC energies in a nonequilibrium-statistical framework [4, 5, 6, 7, 8, I start with $\mathrm{q}=\nu=1$ corresponding to the standard FPE. For a linear drift function

$$
J(y)=\left(y_{e q}-y\right) / \tau_{y}
$$

with the rapidity relaxation time $\tau_{y}$, this is the so-called Uhlenbeck-Ornstein process, applied to the relativistic invariant rapidity. The equilibrium value is $y_{e q}=0$ in the center-of-mass for symmetric systems, whereas $y_{e q}$ is calculated from the given masses and momenta for asymmetric systems. Using $\delta$-function initial conditions at the beam rapidities $\pm y_{b}( \pm 5.36$ at $\mathrm{p}=100 \mathrm{GeV} / \mathrm{c}$ per nucleon $)$, the equation has analytical Gaussian solutions. The mean values shift in time towards the equilibrium value according to

$$
<y_{1,2}(t)>=y_{e q}\left[1-\exp \left(-2 t / \tau_{y}\right)\right] \pm y_{b} \exp \left(-t / \tau_{y}\right)
$$

For a constant diffusion coefficient $D_{y}$, the variances of both distributions have the wellknown simple form

$$
\sigma_{1,2}^{2}(t)=D_{y} \tau_{y}\left[1-\exp \left(-2 t / \tau_{y}\right)\right]
$$

whereas for a time dependent diffusion coefficient $D_{y}(t)$ that accounts for collective (multiparticle) and memory effects the analytical expression for the variances becomes more involved [11]. At short times $t / \tau_{y}<<1$, a statistical description is of limited validity due to the small number of interactions. A kinematical cutoff prevents the diffusion into the unphysical region $|y|>y_{b}$. For larger values of $t / \tau_{y}$, the system comes closer to statistical equilibrium such that the FPE is valid.

Since the equation is linear, a superposition of the distribution functions emerging from $R_{1,2}(y, t=0)=\delta\left(y \mp y_{b}\right)$ yields the exact solution, with the normalization given by 
the total number of net baryons and the value of $t / \tau_{y}$ at the interaction time $\mathrm{t}=\tau_{\text {int }}$ (the final time in the integration of (1)) determined by the peak positions [4]. This approach has also been applied sucessfully to produced particles at RHIC energies [9], although there the initial conditions are less straightforward.

The microscopic physics is contained in the diffusion coefficient. Macroscopically, the transport coefficients are related to each other through the dissipation-fluctuation theorem (Einstein relation) with the equilibrium temperature $\mathrm{T}$

$$
D_{y}=\alpha \cdot T \simeq f\left(\tau_{y}, T\right)
$$

In 4] I have obtained the analytical result for $D_{y}$ as function of $\mathrm{T}$ and $\tau_{y}$ from the condition that the stationary solution of (1) is equated with a Gaussian approximation to the thermal equilibrium distribution in y-space (which is not exactly Gaussian, but very close to it) as

$$
D_{y}\left(\tau_{y}, T\right)=\frac{1}{2 \pi \tau_{y}}\left[c(\sqrt{s}, T) m^{2} T \cdot\left(1+2 \frac{T}{m}+2\left(\frac{T}{m}\right)^{2}\right)\right]^{-2} \exp \left(\frac{2 m}{T}\right)
$$

with $c(\sqrt{s}, T)$ given in $[5$, in closed form. This allows to maintain the linearity of the model and hence, to solve the FPE analytically, although small corrections are to be expected. They cause minor deviations in the calculated rapidity distributions that are within the size of the error bars of the experimental data at SPS energies.

In the linear model, net baryon rapidity spectra at low SIS-energies are well reproduced, whereas at AGS-, SPS- and RHIC energies I find discrepancies to the data that rise strongly with $\sqrt{s}$. At SPS energies, this has recently been confirmed in a numerical calculation [6, 7] based on a nonlinear drift

$$
J(y)=-\alpha \cdot m_{\perp} \sinh (y) \equiv-\alpha \cdot p_{\|}
$$

with the transverse mass $m_{\perp}=\sqrt{m^{2}+p_{\perp}^{2}}$, and the longitudinal momentum $p_{\|}$. Together with the dissipation-fluctuation theorem (5), this yields exactly the Boltzmann distribution as the stationary solution of (1) for $\nu=q=1$. The corresponding numerical solution with $\delta$-function initial conditions at the beam rapidities is, however, only approximately correct since the superposition principle is not strictly valid for a nonlinear drift. Still, the numerical result shows almost the same large discrepancy between data and theoretical 
rapidity distribution as the linear model. In a $\mathrm{q}=1$ framework, the net proton distribution in $\mathrm{Pb}+\mathrm{Pb}$ at the highest SPS energy requires a rapidity width coefficient $\sqrt{D_{y} \tau_{y}}$ that is enhanced beyond the theoretical value (5) by a factor of $g(\sqrt{s}) \simeq 2.6$ due to memory and collective effects [4, 5, 11, Fig.1.

Alternatively, a transition to nonextensive statistics [10, 12, 13, maintaining the weakcoupling diffusion coefficient from (5) requires a value of $\mathrm{q}$ that is significantly larger than one. In an approximate numerical solution of (1) with the nonlinear drift (7), $\mathrm{q}=1.25$ has been determined for the net-proton rapidity distribution in $\mathrm{Pb}+\mathrm{Pb}$ collisions at the $\mathrm{SPS}$ 6. 7. The only free parameter is $q$, whereas in the linear $q=1$ case the enhancement of $D_{y}$ beyond (5) is the only parameter.

This value of $\mathrm{q}$ in the nonlinear model is considerably larger than the result $q\left(\sqrt{s_{N N}}\right)=1.12$ extrapolated from Wilk et al. [8] at the SPS-energy $\sqrt{s_{N N}}=17.3$ $\mathrm{GeV}$. Here, the relativistic diffusion approach is applied to produced particles in protonantiproton collisions in the energy range $\sqrt{s}=53 \mathrm{GeV}-1800 \mathrm{GeV}$, and used to predict LHC-results. The nonlinearity $q>1$ appears to be an essential feature of the $p \bar{p}$ data. The larger value of $\mathrm{q}$ in heavy systems as compared to $p \bar{p}$ at the same NN-center-of-mass energy emphasizes the increasing superdiffusive effect of multiparticle collisions both between participants, and between participants and produced particles. It is, however, conceivable that both a violation of (5) due to memory effects, and $q>1$ have to be considered in a complete description.

Investigating the $\mathrm{Au}+\mathrm{Au}$ system at $\mathrm{RHIC}$ energies in the linear $\mathrm{q}=1$ model, it is now easy to show that in central collisions (10 per cent of the cross-section) the nonequilibrium net-proton rapidity spectrum calculated with the standard dissipation-fluctuation theorem underpredicts the widths of the nonequilibrium fractions of the experimental distribution 14. 1] significantly. In this comparison, the temperature $\mathrm{T}=170 \mathrm{MeV}$ is taken from a thermal fit of charged antiparticle-to-particle ratios in the $\mathrm{Au}+\mathrm{Au}$ system at $200 \mathrm{GeV}$ per nucleon [16, 15], and the theoretical value of the rapidity width coefficient calculated from the analytical expression (6) is $\sqrt{D_{y} \tau_{y}}=7.6 \cdot 10^{-2}$. As was shown in [6 for SPS results, the discrepancy persists in case of the nonlinear drift (7) that yields the exact Boltzmann-Gibbs equilibrium solution for $\mathrm{q}=1$

$$
E \frac{d^{3} N}{d^{3} p}=\frac{d^{3} N}{d y \cdot m_{\perp} d m_{\perp} d \phi} \propto E \cdot \exp (-E / T) .
$$


An enhancement of the weak-coupling rapidity width coefficient by a factor of $g(\sqrt{s}) \simeq 3.7$ due to collective and memory effects in the system corresponding to a violation of (5) yields a good reproduction of the nonequilibrium contributions with $\tau_{\text {int }} / \tau_{y}=0.26$, but the midrapidity valley that is present in the data is completely absent in the extensive nonequilibrium $\mathrm{q}=1$ case, solid curves in Fig.1 (bottom). This remains true in the nonextensive case $(1<q<1.5)$, with an approximate distribution function [10, 13, 6, 7, 8] that is given by a linear superposition of Tsallis-like power-law solutions of (1)

$$
R_{1,2}(y, t)=\left[1-(1-q) \frac{m_{\perp}}{T} \cosh \left(y-<y_{1,2}(t)>\right)\right]^{\frac{1}{1-q}} .
$$

The dashed curves in Fig.1 show the result for $\mathrm{q}=1.4, \mathrm{~T}=170 \mathrm{MeV}$ and a mean transverse mass $<m_{\perp}>=1.2 \mathrm{GeV}$. This solution is far from the nonextensive equilibrium distribution which would be reached for $\left\langle y_{1,2}(t \rightarrow \infty)\right\rangle=y_{\text {eq }}$, and it is significantly below the midrapidity data. The result is even worse for larger values of $m_{\perp}$. In contrast, the $\mathrm{Pb}+\mathrm{Pb}$ data at SPS energies [20] are well described both in the linear model [4] (Fig.1, top) and in the nonlinear case (cf. [6] for results with a time-dependent temperature and an integration over transverse mass).

It turns out, however, that the RHIC data can be interpreted rather precisely in the linear $\mathrm{q}=1$ framework with the conjecture that a fraction of $Z_{e q} \simeq 22$ net protons near midrapidity reaches local statistical equilibrium in the longitudinal degrees of freedom. The variance of the equilibrium distribution $R_{e q}(y)$ at midrapidity is broadened as compared to the Boltzmann result (dashed curve in Fig.2) due to collective (multiparticle) effects by the same factor that enhances the theoretical weak-coupling diffusion coefficient derived from (5). This may correspond to a longitudinal expansion velocity of the locally equilibrated subsystem as accounted for in hydrodynamical descriptions. In the nonextensive model, the corresponding equilibrium distribution is broadened (blueshifted) according to $q \simeq 1.4$.

Microscopically, the baryon transport over 4-5 units of rapidity to the equilibrated midrapidity region is not only due to hard processes acting on single valence (di)quarks that are described by perturbative QCD, since this yields insufficient stopping. Instead, additional processes such as the nonperturbative gluon junction mechanism [17] are necessary to produce the observed central valley. This may lead to substantial stopping even at LHC energies where the separation of nonequilibrium and equilibrium net baryon 
fractions in rapidity space is expected to be even better than at RHIC. In the late thermalization stage [18, nonperturbative approaches to QCD thermodynamics are expected to be important.

Recent work indicates that one may account for the observed stopping in heavyion collisions at SPS and RHIC energies with string-model parameters determined from hadron-hadron collisions [19]. If this was confirmed, the corresponding rapidity distributions would not be considered to be anomalous from a microscopic point of view. However, this view does not offer a distinction between nonequilibrium and equilibrium contributions to the net baryon rapidity spectra, which both exist at RHIC energies, and are anomalously broadened.

Macroscopically, the complete solution of (1) in the $q=1$ case is a linear superposition of nonequilibrium and equilibrium distributions (Fig.2, bottom)

$$
R\left(y, t=\tau_{\text {int }}\right)=R_{1}\left(y, \tau_{\text {int }}\right)+R_{2}\left(y, \tau_{\text {int }}\right)+R_{e q}(y) .
$$

It yields a good representation of the preliminary BRAHMS data [1]. (In the $q>1$ case, the corresponding solution is questionable because the superposition principle is violated). Based on (10), the transition from net-proton rapidity spectra with a central plateau in $\mathrm{Pb}+\mathrm{Pb}$ at the lower SPS energies [11, via a double-humped distribution at the maximum SPS energy [20, 4, 6, 7, 11] to the central valley at RHIC [1] is well understood. It has not yet been possible to identify a locally equilibrated subsystem of net baryons at midrapidity below RHIC-energies, although it cannot be excluded that it exists. At SPS energies, the data [20] are well described by the nonequilibrium distributions, and it is much more difficult (and probably impossible) to identify a locally equilibrated component because the relevant rapidity region is comparatively small, and an equilibrated contribution cannot be separated from the nonequilibrium components in rapidity space. In $p \bar{p}$-collisions at $\sqrt{s}=53-900 \mathrm{GeV}$, no convincing signatures of a phase transition were found [21].

Most remarkably, Fig. 2 suggests that in central $\mathrm{Au}+\mathrm{Au}$ collisions at $\sqrt{s_{N N}}=200 \mathrm{GeV}$ there is no continuous transition from the nonequilibrium to the equilibrium contribution in net-proton rapidity spectra as function of time. This may well be due to a sudden enhancement in the number of degrees of freedom as encountered in the deconfinement of participant partons, which enforces a very rapid local equilibration in a fraction of the system. The central valley in net-proton rapidity spectra at RHIC energies could thus be 
used as an indicator for partonic processes that lead to a baryon transfer over more than 4 units of rapidity, and for quark-gluon plasma formation.

To conclude, I have interpreted recent results for central $\mathrm{Au}+\mathrm{Au}$ collisions at RHIC energies in a Relativistic Diffusion Model (RDM) for multiparticle interactions based on the interplay of nonequilibrium and equilibrium ("thermal") solutions. In the linear version of the model, analytical results for the rapidity distribution of net protons in central collisions have been obtained. The anomalous enhancement of the diffusion in rapidity space as compared to the expectation from the weak-coupling dissipation-fluctuation theorem due to high-energy multiparticle effects has been discussed using extensive and nonextensive statistics.

A significant fraction of about 14 per cent of the net protons reaches local statistical equilibrium in a fast and discontinuous transition which is likely to indicate parton deconfinement. The precise amount of protons in equilibrium is related to the experimental value of the rapidity density close to $y=0$ and hence, possible changes in the final data will affect the percentage. It has not yet been possible to isolate a corresponding fraction of longitudinally equilibrated net protons in the $\mathrm{Pb}+\mathrm{Pb}$ system at SPS energies. Since no signatures of a transition to the quark-gluon plasma have been observed in $p \bar{p}$-collisions, Quark-Matter formation is clearly a genuine many-body effect occuring only in heavy systems at sufficiently high energy density. Consequently, a detailed investigation of the flat midrapidity valley found at RHIC, and of its energy dependence is very promising. 


\section{References}

[1] J.H. Lee et al., BRAHMS Collaboration, Proc. Quark Matter 2002, Nantes, in press.

[2] K. Adcox et al., PHENIX Collaboration, Phys. Rev. Lett. 89 (2002) 092302.

[3] C. Adler et al., STAR Collaboration, Phys. Rev. Lett. 87 (2001) 262302.

[4] G. Wolschin, Eur. Phys. J. A 5 (1999) 85.

[5] G. Wolschin, Europhys. Lett. 47 (1999) 30.

[6] A. Lavagno, Physica A 305 (2002) 238.

[7] W.M. Alberico, A. Lavagno, and P. Quarati, nucl-th/0205044.

[8] M. Rybczyński, Z. Włodarczyk, and G. Wilk, hep-ph/0206157.

[9] M. Biyajima, M. Ide, T. Mizoguchi, and N. Suzuki, Prog. Theor. Phys. 108 (2002) 559;

M. Biyajima and T. Mizoguchi, Submitted to Prog. Theor. Phys., nucl-th/0209004.

[10] C. Tsallis, J. Stat. Phys. 52 (1988) 479;

C. Tsallis and D.J. Bukman, Phys. Rev. E 54 (1996) R2197.

[11] G. Wolschin, Proc. ICPAQGP, Jaipur (2001), in press.

[12] W.M. Alberico, A. Lavagno, and P. Quarati, Eur. Phys. J. C 12 (2000) 499.

[13] G. Wilk and Z. Włodarczyk, Phys. Rev. Lett. 84 (2000) 2770.

[14] I.G. Bearden et al., BRAHMS Collaboration, Proc. Quark Matter 2002, Nantes, in press.

[15] F. Becattini et al., Phys. Rev. C 64 (2001) 024901.

[16] I.G. Bearden et al., BRAHMS Collaboration, Submitted to Phys. Rev. Lett., nucl-ex/0207006. 
[17] G.C. Rossi and G. Veneziano, Phys. Rep. 63 (1980) 153;

D. Kharzeev, Phys. Lett. B 378 (1996) 238;

S.E. Vance, M. Gyulassy, X.N. Wang, Phys. Lett. B 443 (1998) 45.

[18] J. Berges and J. Cox, Phys. Lett. B 517 (2001) 369 ;

G. Aarts and J. Berges, Phys. Rev. D64 (2001) 105010.

[19] A. Capella, Phys. Lett. B 542 (2002) 65.

[20] H. Appelshäuser et al., NA49 Collaboration, Phys. Rev. Lett. 82 (1999) 2471.

[21] C. Geich-Gimbel, Int. J. Mod. Phys. A4 (1989) 1527. 


\section{Figure captions}

FIG. 1: Nonequilibrium contributions to the net-proton rapidity spectra of $\mathrm{Au}+\mathrm{Au}$ at $\sqrt{s_{N N}}=200 \mathrm{GeV}$ in the Relativistic Diffusion Model (RDM) with an equilibrium temperature of $\mathrm{T}=170 \mathrm{MeV}$ (bottom). The solid curve is obtained in the linear model ( $q=1$ ) with an enhanced width [5, 11] due to multiparticle effects according to the preliminary BRAHMS data [1, squares. The dashed curve corresponds to $\mathrm{q}=1.4$ and $\left\langle m_{\perp}>=1.2 \mathrm{GeV}\right.$ in the nonlinear model. At SPS energies (top), NA49 data 20] for central events ( 5 per cent) including $\Lambda$ feed-down corrections are compared with the linear model [4, 11].

FIG. 2: Net-proton rapidity spectra for central collisions of $\mathrm{Au}+\mathrm{Au}$ at $\sqrt{s_{N N}}=200$ $\mathrm{GeV}$ consist of two nonequilibrium components (solid peaks, top) plus an equilibrium contribution at $\mathrm{T}=170 \mathrm{MeV}$, dashed curve. It is broadened due to collective (multiparticle) effects, shaded area, and after hadronization, it contains $Z_{e q} \simeq 22$ protons. Superposition creates the flat valley near midrapidity (bottom) in agreement with the preliminary BRAHMS data points [1]; diamonds include $\Lambda$ feed-down corrections at $\mathrm{y}=0$ (17.5 per cent) and $\mathrm{y}=2.9$ (20 per cent), respectively. Arrows indicate the beam rapidities $\pm y_{b}$. 


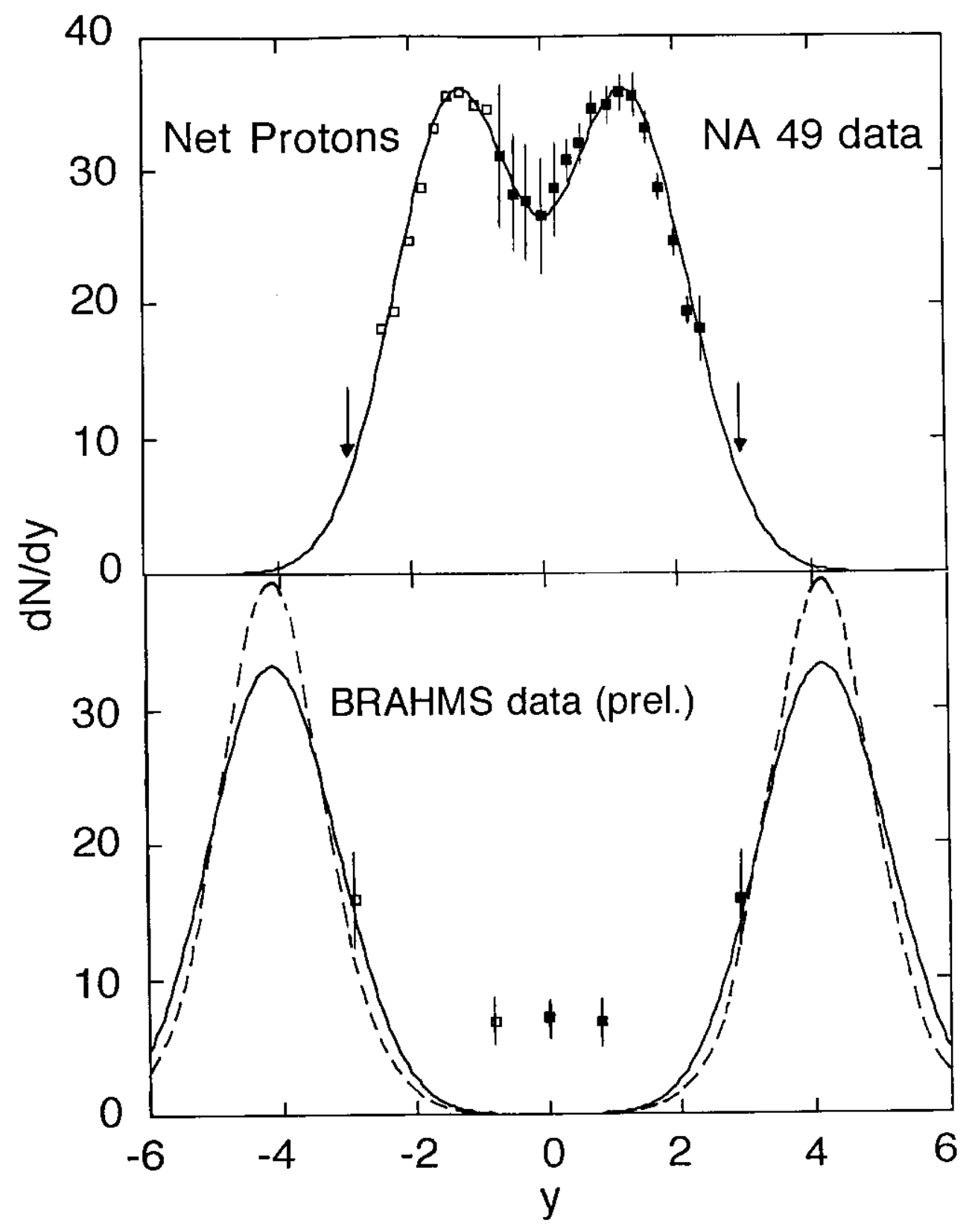

Fig. 1. 


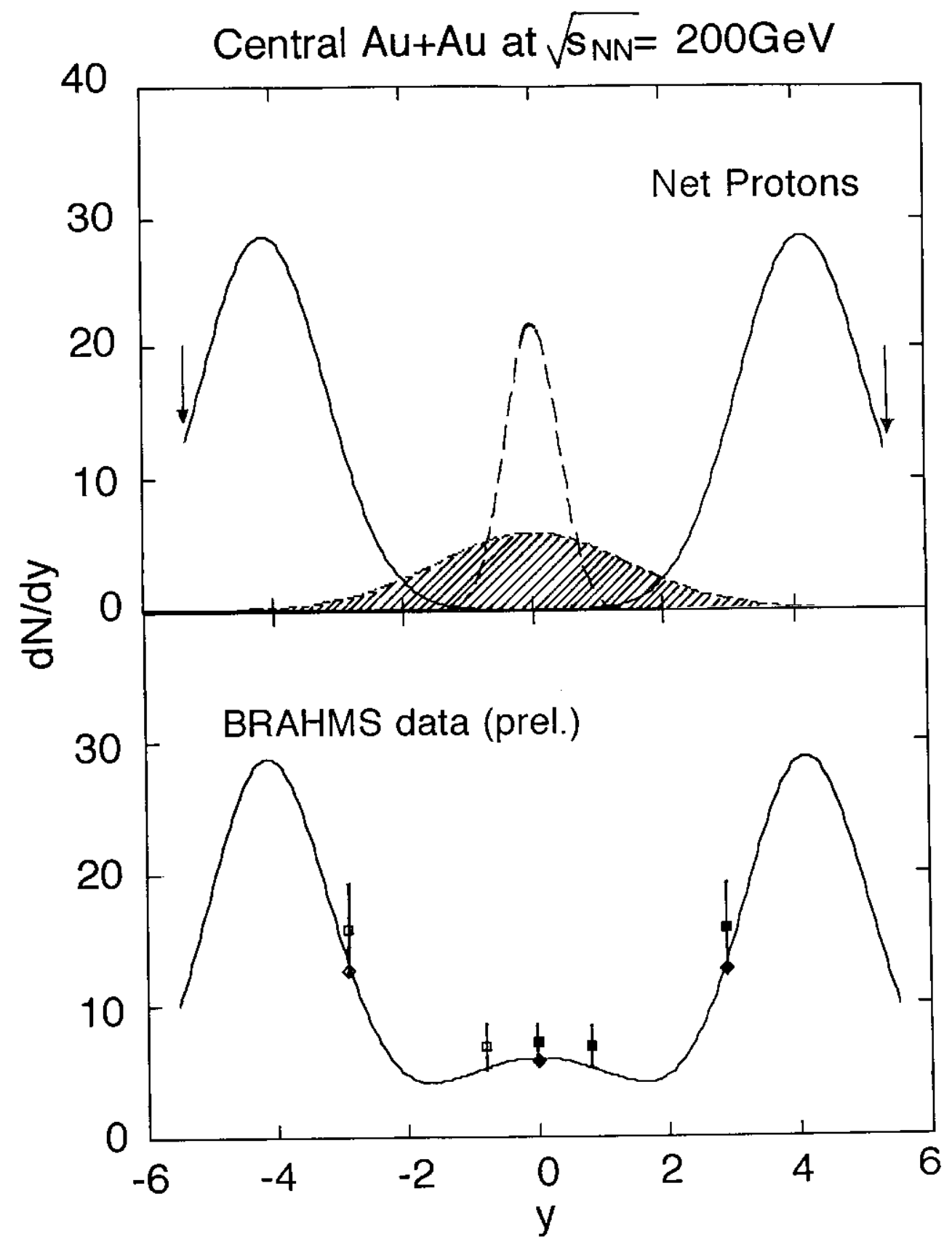

Fig. 2. 